\title{
Unilateral Familial Exudative Vitreoretinopathy: Clinical Profile and Pathology
}

\author{
Nina S. Boal ${ }^{a} \quad$ Alberto G. Distefano ${ }^{a}$ Stephen P. Christiansen ${ }^{a} b^{b}$ \\ Nora V. Laver ${ }^{\mathrm{C}}$ \\ aDepartment of Ophthalmology, Boston Medical Center, Boston University School of \\ Medicine, Boston, MA, USA; bepartment of Pediatric Ophthalmology, Boston Medical \\ Center, Boston, MA, USA; 'Ophthalmic Pathology, Departments of Ophthalmology, \\ Pathology and Laboratory Medicine, Tufts Medical Center and Tufts University School of \\ Medicine, Boston, MA, USA
}

\section{Keywords}

Familial exudative vitreoretinopathy - Pathology of familial exudative vitreoretinopathy . Unilateral retinal dysplasia

\section{Abstract}

We report a case of a newborn with unilateral retinal detachment that could not be repaired. At examination under anesthesia, the retina was markedly abnormal and a presumptive diagnosis of retinal dysplasia was made. Several years later, the eye was enucleated because it was blind and painful. Final pathology was consistent with familial exudative vitreoretinopathy (FEVR). The literature describing unilateral retinal dysplasia is sparse. This case adds to the clinical spectrum of pathologic findings in FEVR.

\section{Introduction}

Familial exudative vitreoretinopathy (FEVR) is a rare inheritable disorder of retinal vascular development. This disease was first described in 1969 by Criswick and Schepens [1] and is typically thought to be bilateral. However, disease asymmetry is typical and variable phenotypic expressivity of retinal abnormalities may result in unilateral FEVR [2-6]. FEVR may masquerade as other retinal disorders as its features range from avascularity of the peripheral retina to retinal folds and detachments [2]. Since its discovery, several histopathologic descriptions have been published, though none recently [7-9]. We report a case of a 
patient who presented with unilateral retinal dysplasia that ultimately required enucleation. We describe the patient's presentation and histopathologic findings of this case.

\section{Case Report}

A 2-day-old girl was evaluated for a decreased red reflex in the right eye. She was born at term by spontaneous vaginal delivery to a 21-year-old woman who had an uncomplicated pregnancy. There was no family ocular history. On initial examination, pupils were equal, round, and reactive bilaterally with no afferent pupillary defect; however, a right afferent pupillary defect was noted on subsequent examinations. A decreased red reflex was noted in the right eye. Slit lamp examination of the right eye revealed a posterior lenticular membrane with a small area of blood adherent to the lens capsule. The left eye was unremarkable. Dilated funduscopic examination of the right eye showed dense intravitreal hemorrhage and a limited view of the retina. The left eye was remarkable for a small temporal intraretinal hemorrhage but no other peripheral retinal abnormalities such as avascularity, neovascularization, or exudates.

At 3 weeks old, the patient underwent examination under anesthesia which revealed a retrolental collection of dehemoglobinized and fresh blood in the right eye. Corneal diameters were symmetric, $10 \mathrm{~mm}$ in both eyes. Dilated fundoscopic examination of both eyes was unchanged. B-scan ultrasonography of the right eye confirmed a mobile vitreous hemorrhage with no obvious stalk and a few areas of high internal reflectivity corresponding with a possible retinal detachment; however, no masses were identified (Fig. 1a). Given the lack of peripheral retinal abnormalities seen on fundoscopic examination in the unaffected left eye, further testing such as fluorescein angiography was not performed. After the examination

Fig. 1. a B-scan ultrasonography of the right eye at age 1 month showed a mobile vitreous hemorrhage with no obvious stalk and high reflective signal on A-scan corresponding with possible retinal detachment; no masses were appreciated. b B-scan ultrasonography of the right eye when the patient was 4 months old shows a $4.1 \times 4.7 \mathrm{~mm}$ well-demarcated, round mass over the optic nerve with high internal reflectivity but no frank calcifications.
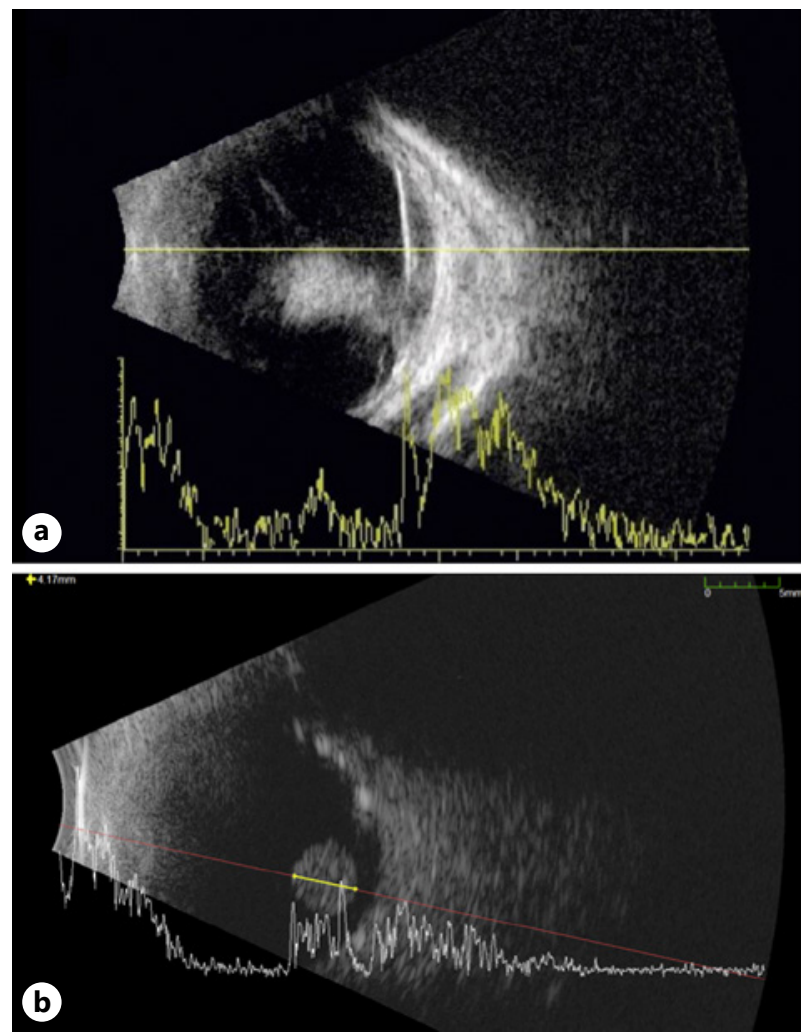
under anesthesia, lensectomy and vitrectomy were performed in the right eye clearing the vitreous hemorrhage. There was a $360^{\circ}$ tractional detachment of the retina in a funnel configuration surrounding a hypoplastic optic nerve. The visible areas of retina were atrophic and showed no retinal vessels or recognizable retinal structures, and repair was not deemed possible. No further intervention was performed.

One month later, the infant returned with an inflamed right eye. On examination, her right eye intraocular pressure was $24 \mathrm{~mm} \mathrm{Hg}$ with a 1-mm layered hyphema. She was managed with prednisolone, atropine, latanoprost, and timolol. At subsequent visits, her right eye was noted to have no light perception, persistently elevated eye pressures to as high as $46 \mathrm{~mm} \mathrm{Hg}$, a chronic hyphema, and corneal blood staining. The mother declined further surgical intervention but agreed to continued observation. Four months later, B-scan ultrasonography of the right eye showed a $4.1 \times 4.7 \mathrm{~mm}$, well-demarcated, round mass over the optic nerve with high internal reflectivity and without any frank calcifications (Fig. 1b). The mass was thought to represent residual retinal dysplasia, but retinoblastoma could not be ruled out. CT and MRI imaging showed a well-demarcated spherical mass over the right optic nerve head. Retinoblastoma could not be ruled out. Enucleation of the right eye was recommended; however, the patient's mother declined surgery.

When the patient was 4 years old, her right eye became more red and painful. External examination of the right eye was notable for ptosis, band keratopathy, and chronic hyphema covering $50 \%$ of the anterior chamber. The mother agreed to proceed with right eye enucleation with dermis fat graft and temporary tarsorrhaphy. Surgery proceeded without complication. Postoperatively, the patient healed well and was fitted for a prosthetic eye. The patient was never found to have any other medical conditions and met all her developmental milestones.

Pathology revealed a completely detached, folded retina with atrophy and gliosis (Fig. 2a). The anterior chamber angle was closed as a result of iris neovascularization and peripheral anterior synechia. Additionally, there was posterior synechiae with seclusio pupillae (Fig. 2b). There was fibrous metaplasia with focal areas of ossification of the retinal pigment epithelium present. The retina showed prominent abnormal intraretinal vascular proliferations in the folded detached retina (Fig. 3a). The atrophic retina showed areas of infolding and dysplasia in the area of detachment (Fig. 3b, c). Additionally, a preretinal fibrovascular membrane was also present (Fig. 3d). Remnants of lens capsule and cataractous lens were present. There was no evidence of persistent fetal vasculature or a posterior lenticular capsular plaque. The optic

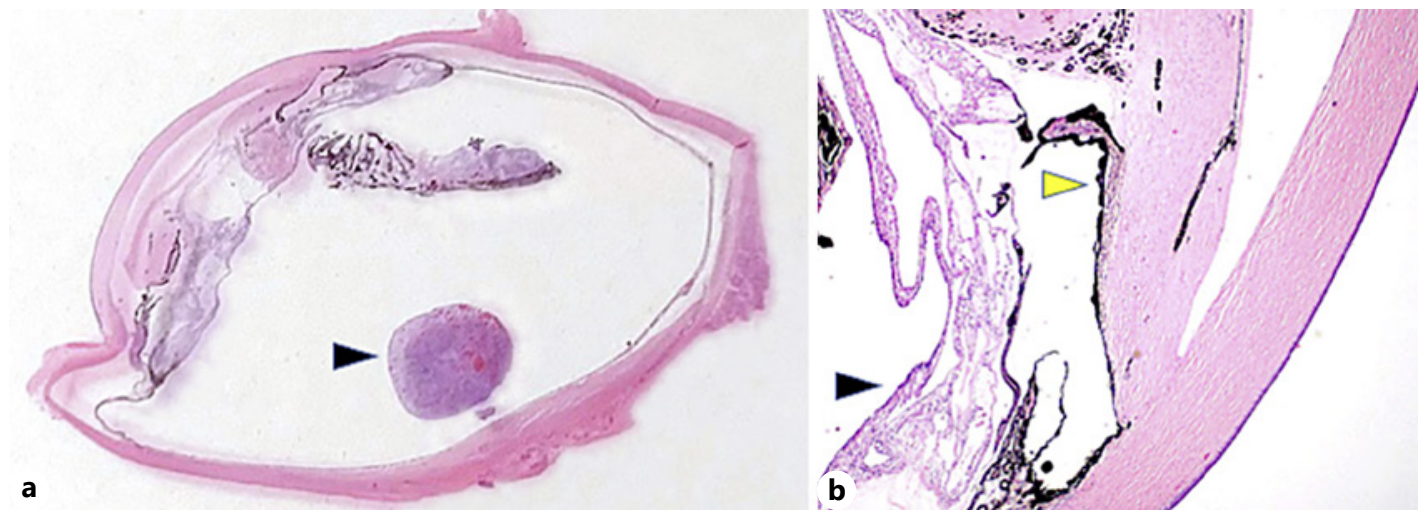

Fig. 2. a Low-power microscopic view of the enucleated right eye with a round mass of detached retina (black arrowhead) (H\&E. $\times 1$ ). b Higher-power view of closed angle with anterior and posterior synechiae and entrapped iris (yellow arrowhead) and detached retina (black arrowhead) (H\&E. ×4). 

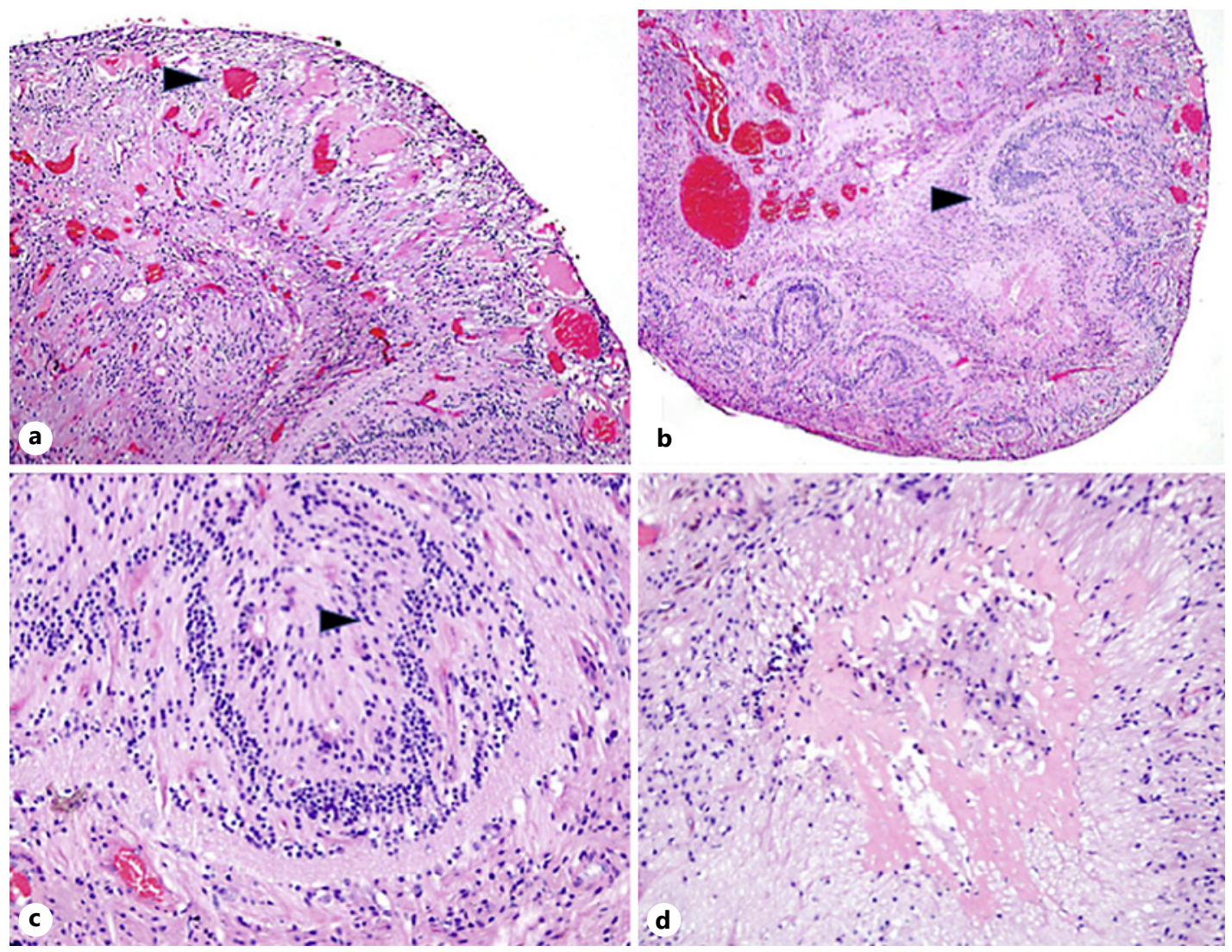

Fig. 3. Higher-power views of the round mass of detached retina. a Prominent abnormal intraretinal vascular proliferations within folded detached retina (H\&E. $\times 10$ ). b, c Atrophic retina with prominent retinal infoldings and dysplasia (black arrowheads) (H\&E. $\times 10$ (b); $\times 20$ (c)). d Preretinal fibrovascular membrane (H\&E. $\times 10)$.

nerve showed atrophic changes secondary to marked retinal atrophy and gliosis. There were no subretinal exudates seen. The overall findings of congenital retinal detachment with a retinal fold were consistent with FEVR.

\section{Discussion}

FEVR is an inherited vitreoretinal disease characterized by abnormal retinal vascular development leading to varying degrees of ischemia and a resultant range of features including retinal folds, hemorrhage, vessel dragging, retinal detachments (RD), and neovascularization [2]. There are currently 5 known FEVR targeted genes (LRP5, FZD4, ZNF408, NDP, and TSPAN12). Reportedly, mutations in these genes account for approximately $50 \%$ of cases [2]. Inheritance may be autosomal dominant, autosomal recessive, or X-linked. Our patient has no family history of FEVR, and molecular testing was not accessible to our patient or her family. Dilated fundoscopic examination of the patient's mother revealed no retinal pathology, and the patient's father was unable to be examined. Patients can present at any age depending on the severity of the disease, but a critical feature is the lack of prematurity. Asymmetric disease is not uncommon in FEVR; patients may have mild or no disease in 1 eye with severe disease in the fellow eye [3-5]. RD in FEVR can be rhegmatogenous, tractional, or serous and are reportedly found in $21-64 \%$ of individuals [3-6]. As was seen in our patient, when 
advanced tractional RD progresses to form a radial fold in the retina, it represents one of the classic features of severe FEVR, described as a "falciform" variant (Fig. 2b, 3a, b) [7-9]. In FEVR, peripheral avascular retina results in retinal ischemia, neovascularization, and ultimately retinal detachment. The degree of retinal avascularity is highly variable and in our patient was severe. While the optic nerve hypoplasia noted in this case is not classically seen in FEVR, we theorize it is not unexpected given our patient's prenatal total retinal detachment. Histopathologic findings in prior reported cases include similar changes as the ones described in this report: retinal detachment, fibrovascular membranes, and intraretinal and subretinal exudates [7-9]. To our knowledge, no published cases of a detached retina tightly folded into a mass in the eye have been published.

Other causes of congenital or early infantile retinal detachment that were considered included retinopathy of prematurity, Norrie disease, incontinentia pigmenti, persistent fetal vasculature, perinatal birth injury, and nonaccidental trauma. Though it was not possible to confirm the diagnosis of FEVR with positive genetic testing, family history, or clinical evidence of FEVR in the fellow eye, the authors felt the atypical presentation and pathologic findings were mostly characteristic of unilateral FEVR.

The patient is currently doing well with a prosthesis in place after her right eye enucleation. The left eye has had no evidence of retinal abnormalities to date.

\section{Statement of Ethics}

Written informed consent was obtained from the patient's mother for publication of this case report and any accompanying images. In accordance with local/national guidelines, the need for ethical approval was not required by the Boston University's IRB committee. All procedures followed were in accordance with the ethical standards and with the Helsinki Declaration.

\section{Conflict of Interest Statement}

The authors declare that they have no conflicts of interest or financial interests to disclose.

\section{Funding Sources}

Authors did not receive any funding for this research study.

\section{Author Contributions}

N.B., N.L., and S.C. were involved in the conception and design of the study. N.B. was involved in drafting the manuscript. N.L. performed the histopathologic examination, interpretation, and documentation of the enucleated eye. All authors were major contributors in revising and approving the final manuscript to be published.

\section{Karger' ${ }^{\prime \prime}$}




\section{References}

1 Criswick VG, Schepens CL. Familial exudative vitreoretinopathy. Am J Ophthalmol. 1969;68(4):578-94.

2 Gilmour DF. Familial exudative vitreoretinopathy and related retinopathies. Eye. 2015;29(1):1-14.

3 Tian T, Chen C, Zhang X, Zhang Q, Zhao P. Clinical and genetic features of familial exudative vitreoretinopathy with only-unilateral abnormalities in a Chinese cohort. JAMA Ophthalmol. 2019;137(9):1054-8.

4 Ranchod TM, Ho LY, Drenser KA, Capone A Jr, Trese MT. Clinical presentation of familial exudative vitreoretinopathy. Ophthalmology. 2011;118(10):2070-5.

5 Shukla D, Singh J, Sudheer G, Soman M, John RK, Ramasamy K, et al. Familial exudative vitreoretinopathy (FEVR). Clinical profile and management. Indian J Ophthalmol. 2003;51(4):323-8.

6 Benson WE. Familial exudative vitreoretinopathy. Trans Am Ophthalmol Soc. 1995;93:473-521.

7 Nishimura M, Yamana T, Sugino M, Kohno T, Yamana Y, Minei M, et al. Falciform retinal fold as sign of familial exudative vitreoretinopathy. Jpn J Ophthalmol. 1983;27(1):40-53.

8 Brockhurst RJ, Albert DM, Zakov ZN. Pathologic findings in familial exudative vitreoretinopathy. Arch Ophthalmol. 1981;99(12):2143-6.

9 Boldrey EE, Egbert P, Gass JD, Friberg T. The histopathology of familial exudative vitreoretinopathy. A report of two cases. Arch Ophthalmol. 1985;103(2):238-41. 